\title{
BMJ Open Patients' and therapists' experiences with a new treatment programme for eating disorders that combines physical exercise and dietary therapy: the PED-t trial. A qualitative study protocol
}

\author{
Gunn Pettersen, ${ }^{1}$ Jan H Rosenvinge, ${ }^{2}$ Maria Bakland, ${ }^{1}$ Rolf Wynn, ${ }^{3}$ \\ Therese Fostervold Mathisen, ${ }^{4}$ Jorunn Sundgot-Borgen ${ }^{4}$
}

To cite: Pettersen G,

Rosenvinge JH, Bakland M, et al. Patients' and therapists' experiences with a new treatment programme for eating disorders that combines physical exercise and dietary therapy: the PED-t trial. A qualitative study protocol. BMJ Open 2018;8:e018708. doi:10.1136/ bmjopen-2017-018708

- Prepublication history for this paper is available online. To view these files, please visit the journal online (http://dx.doi org/10.1136/bmjopen-2017018708).

Received 19 July 2017 Revised 9 November 2017 Accepted 29 November 2017

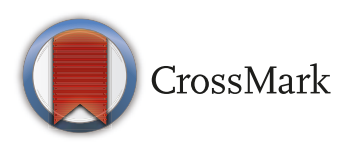

${ }^{1}$ Department of Health and Care Sciences, Faculty of Health Sciences, The Arctic University of Norway, Troms $\emptyset$, Norway ${ }^{2}$ Department of Psychology, Faculty of Health Sciences, UiTThe Arctic University of Norway, Tromsø, Norway

${ }^{3}$ Department of Clinical

Medicine, Faculty of Health

Sciences, UiT-The Arctic

University of Norway, Troms $\emptyset$,

Norway

${ }^{4}$ Department of Sports Medicine, Norwegian School of Sport

Sciences, Oslo, Norway

Correspondence to

Gunn Pettersen;

Gunn.pettersen@uit.no

\section{ABSTRACT}

Introduction Women with bulimia nervosa and binge eating disorder often suffer for many years before they seek professional help. Evidence-based treatments like cognitive-behavioural therapy (CBT) might be poorly accessible, and about $50 \%$ of those who receive CBT respond to it. Such outcome may reflect the heterogeneous nature of eating disorders, and addressing this heterogeneity calls for expanding the portfolio of treatment options. In particular, it is important to explore such options' acceptability, tolerability and affordability expressed through experiences with the treatment. This protocol outlines the rationale and design of a qualitative study. It captures experiences from patients and therapists who were involved in a randomised controlled trial (RCT) exploring the efficacy of a new group-based treatment programme combining physical exercise and dietary therapy.

Methods and analysis 15 patients with bulimia nervosa or binge eating disorder, 10 therapists (physical trainers and dietitians) and 6-10 patients who dropped out of the RCT will be semistructurally interviewed. All interviews will be analysed using a systematic text condensation approach.

Ethics and dissemination Results will be presented in peer-reviewed international journals, and at relevant international conferences. Key findings will be available to study participants as well as to patient organisations and health authorities. The overall study meets the intent and requirements of the Health Research Act and the Declaration of Helsinki. It is approved by the regional committee for medical research ethics (2013/1871). Trial registration number NCT02079935; Pre-results.

\section{INTRODUCTION}

About $3 \%-5 \%$ of the adolescent and adult general population suffers from eating disorders, mostly from bulimia nervosa and binge eating disorder. ${ }^{1}$ Patients with eating disorders are preoccupied with food, eating or not eating and physical appearance. This preoccupation manifests in dieting and

\section{Strengths and limitations of the study}

- The planned study is original as being one of the first to qualitatively investigate patients' and therapists' experiences with a novel treatment for patients with bulimia nervosa and binge eating disorder, where physical exercise and dietary therapy are combined.

- The sample size of $n=35$ in total provides highly transferrable findings.

- Interviews conducted at various time intervals from the treatment study may provide a nuanced picture of perceived benefits.

- The study context may introduce a selection bias if eligible people unfamiliar or uncomfortable with physical activity do not sign up for the study.

often extreme physical exercise behaviour, energy and/or nutritional imbalance as well as behaviours to compensate for overeating. Such behaviour may point to an unhealthy regulation of affects. Eating disorders can be severe and long standing, and the level of severity and duration usually determine the degree of impairment of the sufferers' physical health, cognitive, emotional and interpersonal functioning as well as their quality of life. ${ }^{2}$

The most well-researched treatments for eating disorders are family-based therapy and cognitive-behavioural therapy $(\mathrm{CBT}) .^{23}$ These treatments are generally accepted as evidence based and are recommended in many leading treatment guidelines. ${ }^{4-7}$ Nevertheless, about half of patients admitted to evidence-based treatments respond suboptimally or even fail to respond to them. ${ }^{8}$ One possible reason is that psychological treatments may miss some important maintaining factors, notably those associated with energy imbalance, malnutrition and basic affective 
regulatory mechanisms like excessive exercise. Another reason may be an incomplete knowledge of effectiveness and how patients experience and evaluate treatments. Although some is known about predictors of adherence to manuals of evidence-based treatments ${ }^{9}{ }^{10}$ and challenges in disseminating $\mathrm{CBT},{ }^{11}$ little is known about aspects of therapists' and patients' satisfaction with the available evidence-based treatments.

There is a need to investigate the therapeutic impact of new treatments both in controlled circumstances with selected patients and in actual routine clinical use. A previous randomised controlled trial (RCT) ${ }^{12}$ has provided evidence for guided physical activity as equal to $\mathrm{CBT}$ in reducing eating disorder symptoms, where the physical activity may serve the purpose of regulating negative affects, and promote a positive body image. A more extensive ongoing $\mathrm{RCT}^{13}$ expands on the preliminary one ${ }^{12}$ by exploring the efficacy of a novel treatment approach combining physical exercise and dietary therapy (the PED-t) to enable patients' functional coping and self-regulative activities. The other expansion from the originating study ${ }^{12}$ is the inclusion of qualitative and experiential data from patients and therapists in the ongoing RCT. ${ }^{13}$ Such data may provide information needed to evaluate the efficacy and effectiveness of a specific treatment. Thus, treatment effects may be in vain if for instance, the PED-t is disliked by patients, and for some reason appears to therapists as difficult to provide.

Following the logic of intention-to-treat analyses in RCTs, also experiential analyses need to include experiences from those who drop out from the treatment. This kind of information may capture negative and disadvantageous experiences with the treatment that may be otherwise under-reported. However, little is known about the nature of such experiences.

There is some knowledge about eating disorder patients' generalised treatment experiences gained from case studies, surveys and qualitative studies. This knowledge is confined to therapist qualities, like being skilful and compassionate or to levels of satisfaction when comparing overall modes of treatments, for example, any kind of individual therapy, group therapy or family treatments. ${ }^{14-23}$ Overall, previous findings yield high level of treatment satisfaction, particularly when there is a concordance between initial treatment expectations and outcome satisfaction, ${ }^{24}$ if medical and dietary assistance is provided and if the accessibility of care is high. ${ }^{25-27}$ With respect to patient satisfaction with specific treatment methods or approaches, studies of psychodynamic ${ }^{28}$ and family therapy $^{29}$ have reported rather mixed findings, while cognitive remediation therapy for anorexia nervosa has found high feasibility and acceptability. ${ }^{30}$ Within the realm of the RCT design, favourable experiences have been reported from patients with bulimia nervosa receiving a computer-based $\mathrm{CBT}^{31}$ and from patients with anorexia nervosa receiving two specialist treatment approaches. ${ }^{32}$

Searching the literature, much is written about the risk of therapist fatigue and burnout as well as challenges related to being a therapist, for instance in working with patients with eating disorder. ${ }^{33}$ To the best of our knowledge, far less is known about therapists' experiences with conducting treatment. In many kinds of outpatient psychotherapies, for a wide range of mental disorders, a high level of therapist satisfaction has been reported, ${ }^{34}$ but intriguingly, with a low concordance between therapists' and patients' satisfaction ratings. ${ }^{35}$ Therapist satisfaction has also been related to the use of manualised treatments ${ }^{36}$ and how research protocols are designed in controlled treatment designs, ${ }^{35}$ thus linking satisfaction to adherence and treatment fidelity. Within the eating disorder field, one study ${ }^{37}$ highlighted therapists' satisfaction in running a psychoeducational programme. Within an RCT design, only two studies have explored therapist satisfaction with two approaches to family therapy ${ }^{38}$ and specific specialist treatment approaches for anorexia nervosa. ${ }^{39}$

Aspects of acceptability and tolerability from both those who receive a treatment and those who provide it may help inform treatment development and modifications prior to dissemination to other eating disorder sufferers in other contexts. The dearth of studies of experiential data, especially in controlled trials of novel interventions, is thus surprising. In the present study, we aim to contributing in filling this gap of knowledge. Hence, we address three research questions related to the specific combinatory treatment programme (PED-t) for patients with bulimia nervosa and binge eating disorder provided by therapists in physical exercise and dietary therapy at 6-24 months after the end of the treatment.

1. What are the patients' experiences of the outcome and benefits of the PED-t programme?

2. What are the therapists' experiences of their contribution to the PED-t programme?

3. What are the experiences and explanations from the participants who dropped out of the PED-t programme?

\section{Methods and analysis}

Study context

The context of the current study is the RCT, ${ }^{13}$ which aims to test the efficacy of the combination of guided PED-t versus CBT in treating sufferers from bulimia nervosa and binge eating disorder. Both treatment arms are conducted within a group format of 20 sessions over 16 weeks, and where each group consists of five to eight individuals. Patients who are recruited while a treatment group is running are put on a waiting list control condition of equal length (16 weeks) where they complete all baseline measures.

The RCT excludes men, and female participants with overly manifest comorbid personality disorders and substance abuse, those outside the age range of 18-40 years, as well as those who had received CBT within 2 years before the study start, were pregnant and women with low-weight anorexia nervosa or severe obesity. Those included complete a wide range of biological and 
standardised psychological measures. Details of the RCT protocol have been published elsewhere. ${ }^{13}$

\section{Participant selection and recruitment}

The current interview study comprises three samples.

Sample 1: About 15 participants will be randomly selected among the first 60 participants in the RCT, ${ }^{13}$ and who had completed at least $80 \%$ of the PED-t treatment sessions.

Sample 2: About 6-10 patients who dropped out of the PED-t programme in the time period from completing baseline measurements in the $\mathrm{RCT}^{13}$ to completing $20 \%$ of the treatment sessions will be randomly selected and invited to an interview in order to capture a broader range of treatment-related experiences.

Sample 3: This sample consists of all the 10 therapists who provided the PED-t. They are physical trainers and dietitians at a master level and have completed at least one treatment group.

It is assumed that data saturation will occur in all three subsamples. However, there will be room for flexibility as saturation will be currently evaluated during the data collection period. All participants will receive information by email, outlining the purpose and procedure of the study. In addition to participants' informed consent, a signed letter from the women's general practitioner confirming their suitability for the study is required for final enrolment.

\section{Data collection}

The present study started in 2016 and will end in 2019. The data collection period runs from 2016 to late 2018. Addressing the three research questions, data will be collected through semistructured qualitative interviews and carried out as conversations about the participants' and the therapists' experiences of the PED-t programme. No repeat interviews will be carried out. Interviews will take place at the Norwegian College of Sports Sciences where the RCT study ${ }^{13}$ is conducted or at other locations of the participants' own choice.

The interview guide to address patients' experiences of the outcome and benefits of the PED-t programme (research question 1) is based on relevant literature about eating disorders, the authors' expertise on eating disorders and perspectives from user involvement in the study. This interview guide comprises several questions, for example, what are your overall experiences of participating in the programme related to outcome and benefits? Can you tell whether your daily life has changed in some way during and after the end of the programme? In what way has the programme affected your current attitude and opinion about doing physical activity? In what way has the programme affected your current attitude and opinion about nutrition and addressing nutritional needs? The interview guide to address the therapists' experiences of their contribution to the PED-t programme (research question 2) comprises questions like What are your overall experiences of being a therapist in the programme? Can you explain whether there were aspects of your professional background that stood out as beneficial in conducting the programme? Can you tell whether you experienced incidents or situations in the programme context where your professional knowledge was not sufficient? For the third research question about experiences and explanations of those who dropped out of the PED-t programme only one question will be asked; why did you chose to drop out of the treatment programme? In general, follow-up questions will be freely adjusted to the participant's responses. Each interview will last about 1 hour. All interviews will be audiotaped and then transcribed verbatim and anonymised.

\section{Data analysis and presentation}

The transcribed interviews will be analysed according to the principles of systematic text condensation. ${ }^{40}$ These principles describe a four-step explorative and descriptive method for thematic cross-case analysis of qualitative data. The first step comprise reading and getting an overview of the whole data material of transcribed pages, obtaining an overall impression of experiences. In the second step, units of meaning will be identified, coded and grouped related to the experiences of the study participants. Thirdly, coded data will be condensed, meanings will be abstracted within each of the categories and subcategories and quotes will be selected to illustrate the meaning of each group. In the fourth step, the content and descriptions within each group will be summarised, synthesised and renarrated and finally, the categories will be renamed. The third author will conduct the analysis and the coding, while the other authors will validate the process of systematic text condensation. The consolidated criteria for reporting qualitative research will be used to ensure high-quality qualitative research. ${ }^{42}$

Data will be presented under each category as anonymised quotes, and with acronyms (nicknames) to indicate that the quotes reflect the full range of the sample. In addition, clarity and discussion of minor themes and diverse cases will also be included.

\section{Reflexivity}

A researcher's background and position will affect what they choose to investigate and reflexivity is an attitude of attending systematically to the context of knowledge construction at every step of the research process. ${ }^{40}$ To address the need for reflexivity, the interviewer (MB) will complete a sheet of questions after each interview ${ }^{43}$ about her own emotions and reactions during interview and discuss them with the project group.

\section{User participants' involvement}

To increase the clinical relevance of the research, ${ }^{44}$ an advisory group has been established with members of a national eating disorder patient organisation.

\section{DISCUSSION}

The RCT is expected to yield new knowledge about the efficacy of a new treatment option for eating disorders, that is, the combined guided PED-t programme. It is, 
of course, hoped that it will bring about positive proximal and distal outcomes. The current study is expected to provide an understanding of the effectiveness of the programme in terms of acceptability and tolerance as experienced from both those who provide and those who receive the treatment. An extended source of knowledge about effectiveness will be provided by information from eligible patients who dropped out of the RCT study ${ }^{13}$ from baseline measures and until $20 \%$ of the treatment attendance. In total then, the experiential data will be an important, evidence-based platform for possible adjustments of the new treatment to ease dissemination to patients with eating disorder in other contexts within or outside the healthcare services. In this way, a new treatment programme may be accessible to a larger percentage of sufferers than the relatively low proportion that seek help within the healthcare services, and actually are receiving evidence-based treatments. Moreover, potential findings may open for a debate about which professions that can or should be included in providing help to individuals with eating disorders as well as to other groups of sufferers in society.

Results from this investigation will be presented in peer-reviewed international journals. In addition, major findings will be presented at national and international conferences. Results will be made available to those who participate in the current study as well as to patient organisations and health authorities. In addition, we will bring out major findings to the general public.

Acknowledgements The authors thank the participating patients and therapists for their willingness to share their experiences with the treatment studied.

Contributors MB is responsible for all data collection and coding. GP, RW, TFM, JHR and JS-B: contributed in the data analyses. All authors have contributed in the current manuscript and have approved the final draft. Some members of the patient organisation "Spisfo" will also participate in all parts of the research project, except for the interviewing.

Funding The publication charges for this article have been funded by a grant from the publication fund of UiT The Arctic University of Norway.

Competing interests None declared.

Patient consent Obtained.

Ethics approval The RCT which the current study is a part of, is approved by the Norwegian Regional Committee for Medical and Health Research Ethics identifier: 2013/1871 on 23 October 2013. This study protocol has also been favourably evaluated by the Norwegian Research Council, reference 262184/ MLMT/1512216. Principles of the Declaration of Helsinki will be followed with respect to informed consent and the possibility to unconditionally withdraw from the study on request. The personal identification numbers are stored electronically and separated from the interview transcripts. Transcripts will be kept securely and confidentially and accessible only for the research team. The list that maps ID numbers to the personal information will be erased when the project is finished, thus completely de-identifying the data. All participants will be assured of their anonymity and that the purpose of the study is to learn from their experiences and possibly bring new qualitative knowledge into the field of eating disorder treatment.

Provenance and peer review Not commissioned; externally peer reviewed.

Data sharing statement Data sharing is not applicable to this article as no datasets were generated or analysed. Data are handled and stored according to requirements from the Norwegian Regional Committee for Medical and Health Research Ethics. All data are de-identified. The identification numbers are stored electronically and separated from the clinical data. The list that maps ID numbers to the personal information will be erased when the project is finished, thus completely anonymising the data during the current study. Data will be held within the project group for the future.

Open Access This is an Open Access article distributed in accordance with the Creative Commons Attribution Non Commercial (CC BY-NC 4.0) license, which permits others to distribute, remix, adapt, build upon this work non-commercially, and license their derivative works on different terms, provided the original work is properly cited and the use is non-commercial. See: http://creativecommons.org/ licenses/by-nc/4.0/

(c) Article author(s) (or their employer(s) unless otherwise stated in the text of the article) 2018. All rights reserved. No commercial use is permitted unless otherwise expressly granted.

\section{REFERENCES}

1. Rosenvinge $\mathrm{JH}$, Pettersen $\mathrm{G}$. Epidemiology of eating disorders part II: an update with a special reference to the DSM-5. Adv Eat Disord 2015;3:198-220.

2. Fairburn CG, Harrison PJ. Eating disorders. Lancet 2003;361:407-16.

3. Treasure J. Eating disorders. Lancet 2010;367:1376-7.

4. NICE. Eating disordeders: recognition and treatment. $2017 \mathrm{https}: / /$ www.nice.org.uk/guidance/ng69

5. American Psychiatric Association (APA). Practice guideline for the treatment of patients with eating disorders. Am J Psychiatry 2006;163:4-54

6. The Norwegian Directorate of Health. National guidelines for treating eating disorders. $2017 \mathrm{https}$ //helsedirektoratet.no/retningslinjer/ spiseforstyrrelser

7. Wilson GT, Grilo CM, Vitousek KM. Psychological treatment of eating disorders. Am Psychol 2007;62:199-216.

8. Herpertz S, Hagenah U, Vocks S, et al. The diagnosis and treatment of eating disorders. DeutscheÄrzeblatt Int 2011;108:678-85.

9. Waller $\mathrm{G}$, Stringer $\mathrm{H}$, Meyer $\mathrm{C}$. What cognitive behavioral techniques do therapists report using when delivering cognitive behavioral therapy for the eating disorders? J Consult Clin Psychol 2012;80:171-5.

10. Kosmerly S, Waller G, Lafrance Robinson A. Clinician adherence to guidelines in the delivery of family-based therapy for eating disorders. Int J Eat Disord 2015;48:223-9.

11. Fairburn CG, Wilson GT. The dissemination and implementation of psychological treatments: problems and solutions. Int J Eat Disord 2013;46:516-21.

12. Sundgot-Borgen J, Rosenvinge JH, Bahr R, et al. The effect of exercise, cognitive therapy, and nutritional counseling in treating bulimia nervosa. Med Sci Sports Exerc 2002;34:190-5.

13. Mathisen TF, Rosenvinge JH, Pettersen G, et al. The PED-t trial protocol: The effect of physical exercise -and dietary therapy compared with cognitive behavior therapy in treatment of bulimia nervosa and binge eating disorder. BMC Psychiatry 2017;17:180.

14. Pettersen G, Rosenvinge JH, Wynn R. Eating disorders and psychoeducation-patients' experiences of healing processes. Scand $J$ Caring Sci 2011;25:12-18.

15. Pettersen G, Thune-Larsen KB, Wynn R, et al. Eating disorders: challenges in the later phases of the recovery process: a qualitative study of patients' experiences. Scand J Caring Sci 2013;27:92-8.

16. Beresin EV, Gordon C, Herzog DB. The process of recovering from anorexia nervosa. J Am Acad Psychoanal 1989;17:103-30.

17. Vanderlinden J, Buis $\mathrm{H}$, Pieters $\mathrm{G}$, et al. Which elements in the treatment of eating disorders are necessary 'ingredients' in the recovery process?-A comparison between the patient's and therapist's view. Eur Eat Disord Rev 2007;15:357-65.

18. Clinton D, Björck C, Sohlberg $S$, et al. Patient satisfaction with treatment in eating disorders: cause for complacency or concern? Eur Eat Disord Rev 2004;12:240-6.

19. Rosenvinge $\mathrm{JH}$, Klusmeier AK. Treatment for eating disorders from a patient satisfaction perspective: a Norwegian replication of a British study. Eur Eat Disord Rev 2000;8:293-300.

20. Newton JT, Robinson PH, Hartley P. Treatment for eating disorders in the United Kingdom. Part II. Experiences of treatment: a survey of members of the eating disorders association. Eur Eat Disord Rev 1993;1:10-21.

21. Newton T, Hartley P, Sturmey P. Treatment evaluation for eating disorders by clients with eating disorders. Behavioural Psychotherapy 1993;21:371-4

22. Lkg H, Crisp AH, Callender JS. Recoveryin anorexia nervosa - the patient's perspective. Int J Eat Disord 1992;11:341-50.

23. Bell $L$. What can we learn from consumer studies and qualitative research in the treatment of eating disorders? Eat Weight Disord 2003;8:181-7. 
24. Paulson-Karlsson G, Nevonen L, Engstrom I. Anorexia nervosa: treatment satisfaction. J Fam Ther 2006;28:293-306.

25. Nishizono-Maher A, Escobar-Koch T, Ringwood S, et al. What are the top five essential features of a high quality eating disorder service? A comparison of the views of US and UK eating disorder sufferers, carers and health professionals. Eur Eat Disord Rev 2011;19:411-6.

26. de la Rie S, Noordenbos G, Donker M, et al. Evaluating the treatment of eating disorders from the patient's perspective. Int J Eat Disord 2006;39:667-76

27. Escobar-Koch T, Banker JD, Crow S, et al. Service users' views of eating disorder services: an international comparison. Int $\mathrm{J}$ Eat Disord 2010;43:549-59.

28. Poulsen S, Lunn S, Sandros C. Client experience of psychodynamic psychotherapy for bulimia nervosa: An interview study. Psychother Theory Res Pract 2010;47:469-83.

29. Krautter T, Lock J. Is manualized family-based treatment for adolescent anorexia nervosa acceptable to patients? Patient satisfaction at the end of treatment. J Fam Ther 2004;26:66-82.

30. Money C, Genders R, Treasure J, et al. A brief emotion focused intervention for inpatients with anorexia nervosa: a qualitative study. $J$ Health Psychol 2011;16:947-58.

31. Sánchez-Ortiz VC, House J, Munro C, et al. 'A computer isn't gonna judge you': a qualitative study of users' views of an internetbased cognitive behavioural guided self-care treatment package for bulimia nervosa and related disorders. Eat Weight Disord 2011;16:e93-e101.

32. Lose A, Davies C, Renwick B, et al. Process evaluation of the maudsley model for treatment of adults with anorexia nervosa trial. Part II: Patient experiences of two psychological therapies for treatment of anorexia nervosa. Eur Eat Disord Rev 2014;22:131-9.

33. Thompson-Brenner H, Satir DA, Franko DL, et al. Clinician reactions to patients with eating disorders: a review of the literature. Psychiatr Serv 2012;63:73-8.
34. Puschner B, Bauer S, Kraft S, et al. [Patient and therapist satisfaction in outpatient psychotherapy]. Psychother Psychosom Med Psychol 2005;55:517-26.

35. Chorpita BF, Park A, Tsai K, et al. Balancing effectiveness with responsiveness: therapist satisfaction across different treatment designs in the child STEPs randomized effectiveness trial. $J$ Consult Clin Psychol 2015;83:709-18.

36. Najavits LM, Ghinassi F, Van Horn A, et al. Therapist satisfaction with four manual-based treatments on a national multisite trial: An exploratory study. Psychother Theory Res Pract 2004;41:26-37.

37. Pettersen G, Thune-Larsen KB, Wynn R, et al. Clinical competence and confidence following an interprofessional educational program on eating disorders for nurses and other health care professionals. $J$ Multidiciplin Healthcare 2012;5:201-5.

38. Whitney J, Currin L, Murray J, et al. Family work in anorexia nervosa: a qualitative study of carers' experiences of two methods of family intervention. Eur Eat Disord Rev 2012;20:132-41.

39. Waterman-Collins D, Renwick B, Lose A, et al. Process evaluation of the MOSAIC Trial, Part I: therapist experiences of delivering two psychological therapies for treatment of anorexia nervosa. Eur Eat Disord Rev 2014;22:122-30.

40. Malterud K. Systematic text condensation: a strategy for qualitative analysis. Scand J Public Health 2012;40:795-805.

41. Malterud K. Qualitative research: standards, challenges, and guidelines. Lancet 2001;358:483-8.

42. Tong A, Sainsbury P, Craig J. Consolidated criteria for reporting qualitative research (COREQ): a 32-item checklist for interviews and focus groups. Int J Qual Health Care 2007;19:349-57.

43. Orri M, Sibeoni J, Labey M, et al. Qualitative approach to patientreported outcomes in oncology: protocol of a French study. BMJ Open 2015;5:e008042.

44. Oliver S, Liabo K, Stewart R, et al. Public involvement in research: making sense of the diversity. J Health Serv Res Policy 2015;20:45-51. 